\title{
Amorphous-Crystalline Micro- and Nanostructures in Silicon Fabricated Using Ultrashort Laser Pulses
}

\author{
Yasser Fuentes-Edfuf ${ }^{1, \dagger}$, Mario Garcia-Lechuga ${ }^{1}$, Daniel Puerto ${ }^{1}$, Camilo Florian ${ }^{1}$, Adianez Garcia-Leis ${ }^{2}$, \\ Santiago Sanchez-Cortes ${ }^{2}$, Javier Solis ${ }^{1}$, and Jan Siegel ${ }^{1, *}$ \\ 1 Laser Processing Group, Instituto de Óptica, CSIC, Serrano 121, 28006 Madrid, Spain \\ 2 Instituto de Estructura de la Materia, CSIC, Serrano 121, 28006 Madrid, Spain \\ †.fuentes@io.cfmac.csic.es, ${ }^{+}$j.siegel@csic.es \\ Tel.: +34 915616800, ext. 942322. Fax: +34915645557
}

\begin{abstract}
We demonstrate an innovative way to fabricate different types of amorphous-crystalline surface structures in silicon using ultrashort laser pulses. Fluence-dependent solidification dynamics and interference of incident and scattered laser light are identified as underlying mechanisms.

OCIS codes: (140.3390) Laser materials processing; (320.7090) Ultrafast lasers; (240.3695) Linear and nonlinear light scattering from surfaces; (130.5990) Semiconductors
\end{abstract}

Since their discovery more than three decades ago, laser induced periodic surface structures (LIPSS) have been produced in almost all materials [1]. Upon irradiation with a high intensity laser beam, the material develops selforganized ablated regions in the form of parallel lines which orientation is well defined respect to the polarization of the incident laser beam. However, it has recently been demonstrated that is possible to produce non-ablative LIPSS alternating (a) amorphous and (c) crystalline regions in crystalline Si. [2]. Several semiconductors, including silicon, can re-solidify either in a- or c-phase upon laser-induced melting [3]. By using this phenomenon, extended areas of parallel, a- c-fringes could be written by scanning the sample surface, setting a proper laser fluence, repetition rate, and scan speed [2].

In the present work we identify and investigate additional parameters to control the formation of a- c-structures on Si wafers upon irradiation with multiple femtosecond laser pulses. We have investigated the material response upon static and dynamic (moving sample) irradiation conditions. In the former, surface-depressed annular a-phase rings with a non-depressed crystalline central disk are produced at low fluence and few incident laser pulses (Figure 1a). The formation of a- c-LIPSS takes place when increasing the fluence or the number of pulses, featuring only moderate regularity (Figure 1b). Upon dynamic irradiation, LIPSS become highly periodic and spatially homogeneous. We show that their optical properties, thickness, and lateral dimensions can be defined by adjusting the experimental parameters.

The period of LIPSS structures can be controlled using oblique incidence with two possible periods for ppolarized radiation, derived from a purely geometrical scattering model; $\Lambda^{s-}=\lambda /(1+\sin \theta)$ and $\Lambda^{s+}=\lambda /(1-\sin \theta), \lambda$ being the central laser wavelength and $\theta$ the angle incidence of the laser with respect of the surface normal [1]. 
While both periods inevitably compete in LIPSS formed upon static irradiation, we show that an adequate choice of the scan direction enables a preferential selection of the period to be imprinted (Figure 1c-d) [4]. The simplicity of the fabrication approach combined with the fact that features size and optical properties of the structures can be easily tailored, makes these structures promising for applications in fields including optics and nano-electronics, among others.
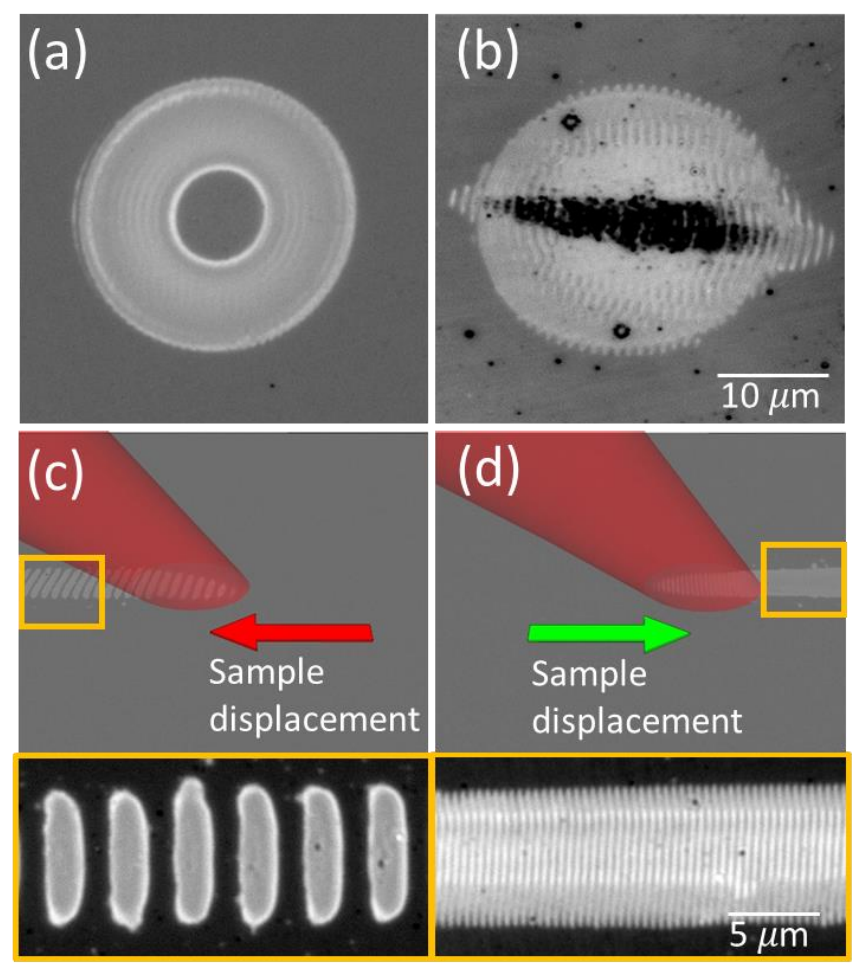

Figure $1(\mathrm{a}, \mathrm{b})$ Optical micrographs of annular structures fabricated in Si upon irradiation with (a) $\mathrm{N}=10, \mathrm{~F}=250 \mathrm{~mJ} / \mathrm{cm}^{2}$ (b) $\mathrm{N}=20, \mathrm{~F}=270 \mathrm{~mJ} / \mathrm{cm}^{2}$, laser pulses at normal incidence $\left(\theta=0^{\circ}\right)$, using a Gaussian intensity distribution with a spot size $d x=d y=59 \mu \mathrm{m}$. (c, d) Sketches of the proposed strategies to write preferentially (c) $\Lambda^{\text {st }}$ or (d) $\Lambda^{\text {s- }}$ LIPSS based only on selecting a determined scan direction. Optical micrographs of the corresponding structures (bottom images). Experimental parameters: $\theta=52^{\circ}, \mathrm{F}=145$ $\mathrm{mJ} / \mathrm{cm}^{2}, \mathrm{v}=220 \mu \mathrm{m} / \mathrm{s}$.

\section{References}

[1] Young, J. F., Preston, J. S., van Driel, H. M., and Sipe, J. E. "Laser-induced periodic surface structure. II. Experiments on Ge, Si, Al, and brass," Phys. Rev. B 27, 1155-1172 (1983).

[2] Puerto, D., Garcia-Lechuga, M., Hernandez-Rueda, J., Solis, J., and Siegel, J. "Femtosecond laser-controlled self-assembly of amorphouscrystalline nanogratings in silicon," Nanotechnology 27, 265602 (2016).

[3] Liu, P.L, Yen, R., Bloembergen, N., and Hodgson, R. T. "Picosecond laser-induced melting and resolidification morphology on Si," Appl. Phys. Lett. 34, 864-866 (1979).

[4] Garcia-Lechuga, M., Puerto, D., Fuentes-Edfuf, Y., Solis, J., and Siegel, J. "Ultrafast Moving-Spot Microscopy: Birth and Growth of LaserInduced Periodic Surface Structures,” ACS Photonics, ACS Photonics 3, 1961-1967 (2016). 